\title{
Efeitos do treinamento aeróbio sobre a capacidade muscular e funcional em pacientes com diabetes mellitus tipo 2
}

\author{
Effects of aerobic training on muscle and functional capacity in patients with \\ type 2 diabetes mellitus
}

\author{
Janaína Lis Sfalcin¹, Paula Caitano Fontela², Eliane Roseli Winkelmann³
}

\begin{abstract}
RESUMO
Introdução: A diabetes mellitus leva a redução da capacidade aeróbia, assim como a diminuição de força muscular respiratória.

Objetivo: Avaliar o impacto do treinamento aeróbio (TA) sobre a força muscular respiratória (FMR) avaliado pela pressão inspiratória (PImáx) e expiratória (PEmáx) máximas, expansibilidade torácica (ExT), capacidade funcional submáxima pelo teste de caminhada de seis minutos (TC6min), resistência muscular localizada (RML) dos membros inferiores e flexibilidade em indivíduos com diabetes mellitus tipo 2 (DM2).

Materiais e Métodos: Ensaio prospectivo, não randomizado com 27 indivíduos com DM2, os quais foram divididos em grupo treinamento aeróbio (G1) e grupo controle (G2) e avaliada pré e pós intervenção a FMR, a ExT, TC6min, RML dos membros inferiores e flexibilidade. O TA durou 8 semanas, três vezes semanais de 60 minutos cada. A prescrição do TA foi realizada a 60 a $70 \%$ da frequência cardíaca máxima monitorado pela escala analógica de Borg.

Resultados: Ocorreu aumento da FMR, 49\% da PImáx e PEmáx, do TC6min, da RML e flexibilidade no grupo G1.

Conclusão: O TA foi efetivo na melhora da força muscular respiratória e capacidade funcional em pacientes com DM2.
\end{abstract}

Palavras-chave: avaliação em saúde; diabetes mellitus tipo 2; tratamento aeróbio.

\begin{abstract}
Introduction: Diabetes Mellitus leads to reduced aerobic capacity as well as the reduction of respiratory muscle strength.

Objective: To evaluate the impact of aerobic training (AT) on respiratory muscle strength (RMS) measured by maximal inspiratory (MIP) and expiratory (MEP) pressures, thoracic expansibility (TEx), submaximal functional capacity (SFC) by 6-min walk test (6MWT) and muscular endurance (ME) of the lower limbs and flexibility in subjects with type 2 diabetes mellitus (T2DM).

Materials and Methods: Prospective trial, non-randomized with 27 subjects with T2DM, which were allocated into AT group (G1) and control group (G2). Evaluations were performed before and after the RMS, TEx, 6MWT, lower limb ME and flexibility interventions. The AT lasted 8 weeks, three times a week, 60 minutes each. The prescription of AT was performed at $60-70 \%$ of maximum heart rate monitored by analog Borg scale.

Results: The study shows that occurred an increase in the RMS (49\% of the MIP and MEP), 6MWT, ME and flexibility in the G1 group.

Conclusion: The TA was effective in improving respiratory muscle strength and functional capacity in patients with T2DM.
\end{abstract}

Keywords: health evaluation; type 2 diabetes mellitus; aerobic treatment.

\footnotetext{
${ }^{1}$ Fisioterapeuta graduada pela Universidade Regional do Noroeste do Estado do Rio Grande do Sul, ljuí, RS, Brasil.

${ }^{2}$ Acadêmica de Fisioterapia do Departamento de Ciências da Vida (DCVida) da Universidade Regional do Noroeste do Estado do Rio Grande do Sul, ljuí, RS, Brasil.

${ }^{3}$ Fisioterapeuta. Doutora em Ciências da Saúde pela UFRGS. Docente do Departamento de Ciências da Vida da Universidade Regional do Noroeste do Estado do Rio Grande do Sul, ljuí, RS, Brasil.
} 


\section{INTRODUÇÃO}

O envelhecimento da população, a urbanização crescente e a adoção de estilos de vida pouco saudáveis estão se tornando os grandes responsáveis pelo aumento da incidência e prevalência da diabetes mellitus. A diabetes mellitus é um conjunto de situações resultantes da incapacidade do organismo em manter o nível de glicose no sangue dentro dos limites normais por deficiência ou ausência total de insulina, manifestando-se por anormalidades no metabolismo dos carboidratos, proteínas e lipídios como também por complicações macrovasculares, microvasculares e neuropáticas ${ }^{1}$. Atualmente, no Brasil existem aproximadamente cinco milhões e meio de diabéticos, sendo $10 \%$ acometidos pela diabetes tipo 1 e $90 \%$ pela tipo $2^{2}$. Mais de $85 \%$ dos óbitos por diabetes ocorrem a partir dos 40 anos de idade, em ambos os sexos ${ }^{3}$.

Os principais efetores da bomba respiratória são os músculos respiratórios, sendo que em patologias que afetam o sistema respiratório, podem ocorrer alterações na função muscular com menor ou maior grau de comprometimento. A diminuição da força muscular respiratória e endurance pode estar implicada como fator da limitação do exercício, diminuição da qualidade de vida e pobre prognóstico, já descrito em outras populações como na insuficiência cardíaca ${ }^{4}$.

O diabético apresenta a capacidade aeróbia mais reduzida que do indivíduo normal ${ }^{1}$ e estudos mais recentes vem mostrando uma diminuição de força muscular respiratória ${ }^{5}$. Por outro lado, observa-se que este comportamento também é verificado em outros indivíduos com ICC ${ }^{6}$, DPOC ${ }^{7}$, distrofias musculares, etc. A explicação fisiopatológica deste comportamento ainda não está bem elucidada na literatura, porém vários estudos foram realizados sobre treinamento específico da musculatura respiratória, mostrando a eficácia deste tipo de intervenção em várias dimensões como qualidade de vida, capacidade funcional, força e resistência muscular respiratória ${ }^{6}$, embora ainda existam poucos estudos com indivíduos diabéticos ${ }^{6}$.

Por sua vez, diversos autores relatam os vários benefícios que o treinamento aeróbio proporciona, tais como a melhora da oxidação dos carboidratos, aumento da densidade capilar do músculo esquelético, redução de peso, auxílio no controle metabólico, melhora da capacidade cardiopulmonar, entre outros benefícios ${ }^{1,6}$. Porém ainda não se sabe a relação da eficácia do treinamento aeróbio sobre a força muscular respiratória no indivíduo diabético. Portanto, há necessidade de estudos que possam mostrar os efeitos deste tratamento sobre o desempenho da força muscular respiratória. Desta forma, este estudo se propôs a verificar o efeito do treinamento aeróbio em indivíduos com diabetes mellitus tipo 2, sob os aspectos da força muscular respiratória (FMR), expansibilidade torácica (ExT), capacidade funcional submáxima (CFS), resistência muscular localizada (RML) dos membros inferiores e flexibilidade.

\section{MATERIAIS E MÉTODOS}

Foi realizado um ensaio clínico prospectivo, não randomizado, em pacientes com diagnóstico de diabetes mellitus tipo 2, que foram recrutados de um grupo de diabéticos e hipertensos da Unidade Básica de Saúde (UBS) no município de ljuí/RS. A seleção dos pacientes diabéticos foi realizada a partir do diagnóstico médico da UBS realizado pelo nível de glicose sanguínea dos indivíduos.

Dentre os critérios de inclusão da amostra foram considerados elegíveis para o estudo os indivíduos com diabetes mellitus tipo 2, faixa etária de 45 a 80 anos, em condições de realizar os testes e o treinamento físico, estabilidade clínica, incluindo não alterar as medicações por um período de dois meses. Foram excluídos os indivíduos com doenças cardíacas e/ou respiratórias, tabagistas ou ex-tabagistas por menos de seis meses, história de acidente vascular encefálico (AVE), acamado, e/ou com dificuldade de deambular, dificuldade de entendimento quanto ao procedimento dos testes, uso de corticóides.

O estudo foi projetado de acordo com Diretrizes e Normas regulamentadoras de Pesquisas Envolvendo Seres Humanos segundo a Resolução do Conselho Nacional de Saúde (CNS) n. 466/12, sendo submetido e aprovado pelo Comitê de Ética e Pesquisa da Universidade Regional do Noroeste do Estado do Rio Grande do Sul - UNIJUÍ, sob protocolo n 057/07. Todos os pacientes foram esclarecidos sobre o projeto e 
assinaram um termo de consentimento informado.

Os indivíduos que foram elegíveis para o estudo foram avaliados por sua história clínica e exame físico. Após os mesmos foram alocados no grupo treinamento aeróbio (G1) ou para o grupo controle (G2). O tipo de estudo foi não randomizado, sendo que o critério de seleção da alocação dos indivíduos foi por livre escolha de acordo com a disponibilidade de tempo para a participação do programa de treinamento aeróbio. Antes e após a intervenção foram realizadas avaliações específicas como os testes da força muscular respiratória, expansibilidade torácica, capacidade funcional submáxima, resistência muscular localizada e flexibilidade dos membros inferiores. O grupo controle não recebeu nenhuma intervenção física somente foi avaliado e após oito semanas foi reavaliado através dos mesmos testes aplicados no grupo que realizou o treinamento aeróbio.

O treinamento aeróbio foi realizado em esteira ergométrica (Movement ${ }^{\circledR}$ - LX 160, Brasil) três vezes por semana, durante oito semanas, totalizando vinte e quatro sessões. O tempo inicial foi de 20 minutos (min) com aumento gradual de $5 \mathrm{~min}$ em cada semana, totalizando $55 \mathrm{~min}$ de treinamento aeróbio. A intensidade do exercício foi prescrita de 60 a 70\% da frequência cardíaca máxima (FCmáx) obtida pela fórmula $F C m a ́ x ~=220$-idade, sendo monitorada pela escala de esforço percebido de Borg correspondendo ao nível moderado a pouco intenso ${ }^{7}$. A escolha dessa forma de prescrição ocorreu porque outros métodos mais apropriados ou recomendados, como obtenção do consumo de oxigênio máximo $\left(\mathrm{VO}_{2} \mathrm{máx}\right)$ ou frequência cardíaca máxima (FCmáx) obtida pelo teste máximo, não estavam disponíveis.

Em cada sessão, inicialmente, foi verificado a pressão arterial (PA) e frequência cardíaca (FC) em repouso (os pacientes deveriam ficar sentados por no mínimo 5 minutos para serem verificados os sinais vitais). Após foi realizado uma série de alongamentos gerais para membros superiores e inferiores e em seguida foi dado início ao programa de exercício aeróbio de acordo com o tempo estipulado para aquela sessão. Antes de iniciar o condicionamento e após finalizá-lo foi realizado um período de aquecimento e desaquecimento de $5 \mathrm{~min}$, respectivamente através do aumento ou redução progressiva da velocidade da esteira. Durante o exercício aeróbio foram verificadas a FC e a percepção de esforço percebido através da escala de Borg ${ }^{7}$ a cada 5 min e a pressão arterial $10 \mathrm{~min}$ antes de finalizar o treinamento (PA pico). Ao final do exercício foram novamente verificados os sinais vitais de repouso.

Foi avaliado o índice de massa corporal (IMC) que é calculado elevando a estatura ao quadrado e dividindo a massa corporal (em Kg) pela estatura. O peso corporal foi avaliado através de uma balança digital (Toledo®) e a estatura através de um estadiômetro (Toledoß) ${ }^{8}$.

A força muscular inspiratória e expiratória foram aferidas através do manovacuômetro digital que é composto por um transdutor de pressão MVD-300 (Microhard System, Globalmed®, Porto Alegre, Brasil), com capacidade de $\pm 300 \mathrm{cmH}_{2} \mathrm{O}$, foram mensuradas como descrito por Dall'ago et al. ${ }^{6}$

A expansibilidade torácica foi verificada através da cirtometria da caixa torácica nos pontos axilar, mamilar e xifoidiano com o uso de fita métrica não extensível, ambos feitos a partir da capacidade residual funcional e inspiração tranquila, respectivamente, com o indivíduo sentado ${ }^{9}$

A capacidade funcional submáxima foi avaliada através da distância percorrida no Teste de Caminhada de Seis Minutos (TC6min) realizado de acordo com as diretrizes estabelecidas pela American Thoracic Society $(\text { ATS })^{10}$. Os indivíduos relatavam o esforço percebido usando a escala de Borg ${ }^{7}$.

Já para avaliar a resistência muscular localizada, o paciente foi orientado a realizar o teste de sentar e levantar por um minuto, onde deveria sentar-se em uma cadeira com as costas repousando sobre a guarda da cadeira, e logo após levantar-se estendendo os joelhos e na sequência sentar-se novamente tocando as costas na guarda da cadeira, o indivíduo deveria realizar o máximo de repetições em um minuto.

Para avaliar a flexibilidade, foi utilizado o teste de distância dedos-solo, em caso do indivíduo tocar o solo esta distância foi considerada zero, como previamente descrito ${ }^{11}$.

Os dados foram analisados no pacote estatístico Statistical Package for Social Science - SPSS (versão 
TABELA 1 - Resultados dos valores basais dos indivíduos com diabetes mellitus tipo 2 que participaram do treinamento aeróbio (G1) e do grupo controle (G2).

\begin{tabular}{|c|c|c|c|}
\hline & G1 & G2 & $\mathbf{p}$ \\
\hline PImáx $\left(\mathrm{cmH}_{2} \mathrm{O}\right)$ & $85,23 \pm 23,98$ & $96,64 \pm 41,84$ & 0,398 \\
\hline PEmáx $\left(\mathrm{cmH}_{2} \mathrm{O}\right)$ & $90,69 \pm 29,14$ & $96,42 \pm 33,8$ & 0,642 \\
\hline Peso (Kg) & $79,71 \pm 15,22$ & $78,50 \pm 10,87$ & 0,827 \\
\hline $\mathbf{R C} / \mathbf{Q}$ & $0,97 \pm 0,40$ & $0,99 \pm 0,07$ & 0,561 \\
\hline$\neq$ Axilar (cm) & $3,26 \pm 0,78$ & $3,21 \pm 1,12$ & 0,885 \\
\hline \# Mamilar $(\mathrm{cm})$ & $2,5 \pm 2,04$ & $3,07 \pm 1,77$ & 0,444 \\
\hline \# Xifoidiana (cm) & $2,23 \pm 1,92$ & $3,35 \pm 2,53$ & 0,207 \\
\hline TC6min (m) & $444,53 \pm 130,07$ & $343,46 \pm 86,70$ & $0,025^{\star}$ \\
\hline TSL1 $\min \left(n^{\circ}\right.$ repetições) & $21,92 \pm 4,90$ & $18,92 \pm 3,73$ & 0,160 \\
\hline Teste Dedos-solo (cm) & $-9,76 \pm 10,26$ & $-9,50 \pm 8,70$ & 0,095 \\
\hline
\end{tabular}

G1: grupo treinamento aeróbio; G2: grupo controle; PImáx: pressão inspiratória máxima; $\mathrm{PEmáx:} \mathrm{pressão} \mathrm{expiratória} \mathrm{máxima;} \mathrm{cmH}_{2} \mathrm{O}$ : centímetros de água; kg: kilogramas; RC/Q: relação cintura-quadril; \# Axilar: Diferença axilar; \# Mamilar: Diferença mamilar; \# Xifoidiana: Diferença xifoidiana; $\mathrm{cm}$ : centímetros; TC6min: teste de caminhada de seis minutos; TSL1min: teste de sentar e levantar em um minuto; no. número; Dedos-solo: teste de flexibilidade dedos-solo; ${ }^{*} \leq 0,05$ para análise dos valores basais.

16.0, Chicago, IL, EUA). Baseados nos resultados do ensaio de Dall'ago et al. ${ }^{6}$ e assumindo que o TA poderia alterar a PImáx, estimamos que o tamanho da amostra de 15 indivíduos em cada grupo deveria ter um poder estatístico de $80 \%$ para detectar uma diferença de $10 \%$ na distância percorrida no teste de caminhada. Dados descritivos são apresentados como média e desvio padrão. Dados basais foram comparados pelo teste t de Student para variáveis contínuas ou por meio do teste exato de Fischer para variáveis categóricas. Os efeitos das intervenções nas variáveis contínuas foram comparadas pela análise de variância de duas vias para medidas repetidas (ANOVA) e análise post-hoc foi realizada pelo teste de Tukey considerando um $\mathrm{p} \leq 0,05$.

\section{RESULTADOS}

Foram avaliados 70 indivíduos e destes 24 foram excluídos de acordo com os critérios de exclusão, 33 aceitaram participar do estudo, porém 6 iniciaram o programa e desistiram (3 por instabilidade pressórica, 1 por motivo particular, 2 por se negarem a realizar o procedimento de manovacuometria) e 27 finalizaram a pesquisa, sendo destes 13 do grupo em treinamento aeróbio (G1) e 14 do grupo controle (G2).

A idade média do G1 e G2 foi de 57,92 e 65,85 anos, respectivamente. No G1 doze indivíduos eram do gênero masculino e um do feminino e no $G 2$ seis indivíduos eram do gênero masculino e oito do feminino. A tabela 1 mostra valores similares das características basais do grupo treinamento aeróbio e grupo controle em quase todas as variáveis, com exceção da capacidade funcional que iniciou com valores aumentados para o grupo treinamento, comportamento que pode ocorrer devido ao fato do estudo ser não randomizado. Os indivíduos receberam medicações similares e mantiveram o mesmo regime médico durante o período do estudo.

$\mathrm{Na}$ tabela 2 estão apresentados os resultados das avaliações realizadas pré e pós treinamento aeróbio do grupo intervenção e do grupo controle. $\mathrm{Na}$ análise da força muscular respiratória evidencia-se um efeito benéfico após 8 semanas de treinamento aeróbio, tanto para a força muscular inspiratória e expiratória, obtendose um aumento de $49 \%$ tanto para PImáx como para PEmáx, sendo estatisticamente significativo e o aumento progressivo ocorreu principalmente nas quatro primeiras semanas, ao contrário do comportamento observado no grupo controle (figura 1).

Já em relação a expansibilidade torácica observou-se um pequeno aumento, mas este não foi estatisticamente significativo, quando comparado o pré e pós treinamento no G1. O grupo controle não obteve alterações.

Verificou-se que houve uma melhora significativa da capacidade funcional submáxima, evidenciada pelo 
TABELA 2 - Resultados dos valores pré e pós avaliação dos indivíduos com diabetes mellitus tipo 2 que participaram do treinamento aeróbio (G1) e do grupo controle (G2).

\begin{tabular}{|c|c|c|c|c|c|c|c|}
\hline & \multicolumn{2}{|c|}{ G1 } & \multicolumn{2}{|c|}{ G2 } & \multirow{2}{*}{$\frac{\mathbf{p}}{\text { Tempo }}$} & \multirow{2}{*}{$\frac{\mathbf{p}}{\text { Interação }}$} & \multirow{2}{*}{$\frac{\mathbf{p}}{\text { Grupo }}$} \\
\hline & Pré & Pós & Pré & Pós & & & \\
\hline PImáx $\left(\mathrm{cmH}_{2} \mathrm{O}\right)$ & $85,23 \pm 23,98$ & $126,0 \pm 23,29$ & $96,64 \pm 41,84$ & $80,07 \pm 27,39$ & $0,007^{*}$ & $0,001^{*}$ & 0,126 \\
\hline PEmáx $\left(\mathrm{cmH}_{2} \mathrm{O}\right)$ & $90,69 \pm 29,14$ & $130,69 \pm 18,69$ & $96,42 \pm 33,8$ & $92,28 \pm 33,10$ & 0,001 & $0,001^{*}$ & 0,123 \\
\hline \% PImáx & $102,61 \pm 27,51$ & $151,69 \pm 25,6$ & $119,56 \pm 62,10$ & $98,86 \pm 38,54$ & 0,015 & $0,001^{*}$ & 0,245 \\
\hline \% PEmáx & $109,77 \pm 33,12$ & $159,07 \pm 23,85$ & $103 \pm 24,47$ & $100,4 \pm 29,85$ & 0,001 & $0,001^{*}$ & 0,002 \\
\hline Peso (Kg) & $79,71 \pm 15,22$ & $78 \pm 14,66$ & $78,50 \pm 10,87$ & $79,68 \pm 10,23$ & 0,390 & $0,001^{*}$ & 0,954 \\
\hline $\mathrm{RC} / \mathrm{Q}$ & $0,97 \pm 0,40$ & $0,95 \pm 0,005$ & $0,99 \pm 0,07$ & $1,007 \pm 0,07$ & 0,370 & 0,005 & 0,150 \\
\hline$\neq$ Axilar (cm) & $3,26 \pm 0,78$ & $3,61 \pm 1,19$ & $3,21 \pm 1,12$ & $3,00 \pm 1,03$ & 0,761 & 0,204 & 0,335 \\
\hline f Mamilar (cm) & $2,5 \pm 2,04$ & $2,34 \pm 2,17$ & $3,07 \pm 1,77$ & $2,42 \pm 1,22$ & 0,077 & 0,268 & 0,630 \\
\hline \# Xifoidiana (cm) & $2,23 \pm 1,92$ & $2,76 \pm 2,24$ & $3,35 \pm 2,53$ & $3,00 \pm 1,41$ & 0,820 & 0,266 & 0,336 \\
\hline TC6min (m) & $444,53 \pm 130,07$ & $536,69 \pm 56,04$ & $343,46 \pm 86,70$ & $317,6 \pm 72,09$ & $0,032^{*}$ & $0,001^{*}$ & $0,001^{*}$ \\
\hline$\%$ TC6min & $96,61 \pm 20,33$ & $111,15 \pm 17,69$ & $74,71 \pm 20,48$ & $69,29 \pm 16,54$ & $0,045^{\star}$ & $0,005^{*}$ & $0,005^{\star}$ \\
\hline TSL1min ( ${ }^{\circ}$ rep.) & $21,92 \pm 4,90$ & $24,84 \pm 4,23$ & $18,92 \pm 3,73$ & $18,85 \pm 3,73$ & $0,047^{*}$ & $0,038^{*}$ & $0,013^{*}$ \\
\hline TDS (cm) & $-9,76 \pm 10,26$ & $-3,46 \pm 4,62$ & $-9,50 \pm 8,70$ & $-8,92 \pm 7,51$ & 0,076 & $0,036^{*}$ & $0,001^{*}$ \\
\hline
\end{tabular}

G1: grupo treinamento aeróbio; $\mathrm{G} 2$ : grupo controle; PImáx: pressão inspiratória máxima; $\mathrm{PEmáx:} \mathrm{pressão} \mathrm{expiratória} \mathrm{máxima;} \mathrm{cmH}_{2} \mathrm{O}$ : centímetros de água; \% PImáx: percentual atingido a partir do previsto na pressão inspiratória máxima; \% PEmáx: percentual atingido a partir do previsto na pressão expiratória máxima; kg: kilogramas; RC/Q: relação cintura-quadril; \# Axilar: Diferença axilar; \# Mamilar: Diferença mamilar; \# Xifoidiana: Diferença xifoidiana; cm: centímetros; TC6min: teste de caminhada de seis minutos; m: metros; \% TC6min: percentual atingido a partir do previsto no teste de caminhada de seis minutos; TSL1min: teste de sentar e levantar em um minuto; no rep.: número de repetições; TDS: teste de flexibilidade dedos-solo; * $p \leq 0,05$ para análise dos valores tempo de treinamento, interação e de grupo.

aumento da distância percorrida e pela diminuição do esforço percebido pela análise da escala de Borg no final do exercício no G1.

Em relação aos dados da resistência dos membros inferiores, verificada através do teste de sentar e levantar, observou-se que o treinamento teve uma influência positiva, evidenciada pelo aumento no número de repetições, sendo esta significativa estatisticamente e pela diminuição da percepção de cansaço, analisados pela escala de Borg. Já no G2 não se observou alterações.

Em relação a flexibilidade da cadeia muscular posterior verificada através do teste dedo-solo observou-se no grupo G1 uma melhora significativa, já no G2 não ocorreu alterações.

\section{DISCUSSÃo}

Este estudo realizado com indivíduos com diabetes mellitus tipo 2 submetidos ao programa de treinamento aeróbio por um período de oito semanas com frequência semanal de 3 vezes, aumentou a força dos músculos respiratórios, tanto inspiratórios quanto expiratórios, expansibilidade torácica, capacidade funcional submáxima, resistência muscular localizada e flexibilidade dos membros inferiores. Os indivíduos que não realizaram nenhuma intervenção física (grupo controle) não obtiveram alterações sobre os parâmetros avaliados o que mostra que o exercício físico aeróbio auxilia na melhora da força da musculatura ventilatória, além das demais variáveis como a capacidade funcional que já são relatados na literatura.

Vários estudos já foram realizados nesta área e descrevem que ocorre uma redução da força muscular respiratória com a idade ${ }^{10,12}$. Porém esta redução também está relacionada a outras patologias como insuficiência cardíaca congestiva $(\text { ICC })^{6}$ e doença pulmonar obstrutiva crônica (DPOC), bem como, recentemente foi evidenciado em diabéticos ${ }^{6,13}$, embora os mecanismos ainda não estão bem elucidados. Recentemente estudos também foram realizados utilizando um tratamento específico para o fortalecimento muscular inspiratório ${ }^{6}$ mostrando um aumento tanto dos parâmetros da força e endurance muscular respiratória, assim como de outros parâmetros indiretos como a capacidade funcional e a qualidade de vida. Por outro lado, já está bem consagrado na literatura que o treinamento aeróbio melhora os parâmetros hemodinâmicos, o controle da glicose do 


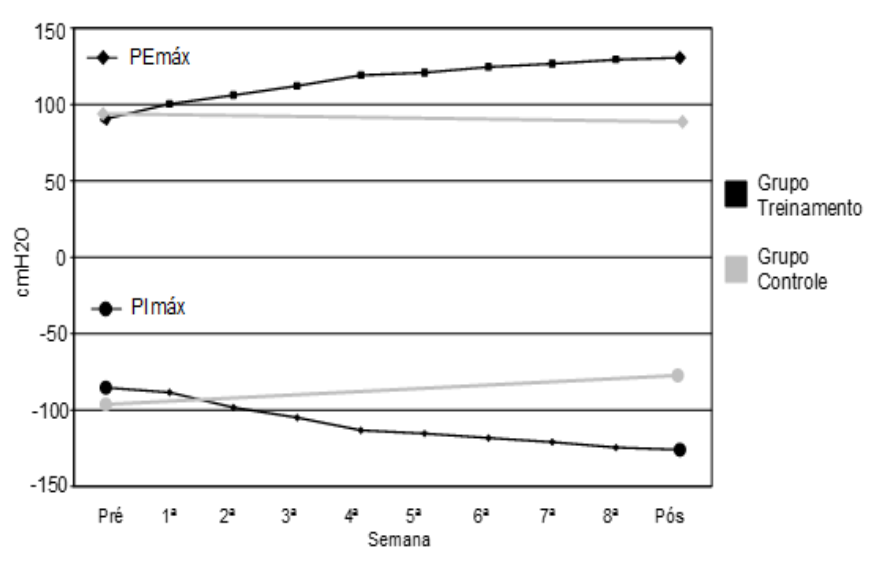

FIGURA 1 - Comportamento da força muscular respiratória dos pacientes diabéticos tipo 2 obtida na avaliação pré e pós no grupo controle e semanalmente no grupo treinamento aeróbio.

sangue, aumento da circulação nas extremidades, reduz o colesterol e os triglicérides, aumenta o colesterol HDL$\mathrm{C}$, controla o peso, melhora o humor, tendo efeitos emocionais benéficos, sobretudo, em casos de depressão ${ }^{14}$. É o tipo de exercício mais realizado no mundo e recomendado tanto para indivíduos saudáveis ou indivíduos com patologias como a hipertensão, diabetes, infarto agudo do miocárdio, ICC dentre outras. Porém, ainda não está bem claro se este tipo de exercício não específico pode melhorar a força muscular respiratória no diabético. Já foi encontrada esta melhora em outras populações como o ICC e aliado a isto foi verificado que o treinamento aeróbio combinado ao treinamento específico exerce melhora adicional nestas variáveis $^{15}$. No presente estudo, verificou-se que o exercício aeróbio exerceu uma influência tanto no aumento da força muscular inspiratória quanto expiratória. O aumento da força foi de $49 \%$, valor este inferior aos achados em outras populações, como em pacientes com $\mathrm{ICC}^{6}$, com tratamento específico. Porém, cabe ressaltar, que os indivíduos da presente investigação não possuíam diminuição de força muscular inspiratória e mesmo assim o exercício aeróbio contribuiu para o acréscimo deste parâmetro. Sendo assim, apresentavam valores normais de força muscular respiratória e mesmo com um treinamento inespecífico foram capazes de aumentar ainda mais estes valores. Isto é diferente dos indivíduos apresentarem redução da força muscular ventilatória e recuperarem posteriormente ao tratamento. Contudo, temos carência na literatura dos efeitos do treinamento aeróbio sobre a musculatura respiratória em pacientes diabéticos com e sem diminuição de força muscular inspiratória.

Foi observado que o treinamento aeróbio exerceu uma influência positiva tanto no incremento da força muscular inspiratória bem como da expiratória, sendo que este aumento foi mais evidente nas primeiras quatro semanas. A glicose absorvida pelo músculo aumenta na medida em que o exercício muscular vai aumentando seu tempo de duração, ou seja, nos primeiros dois minutos de atividade muscular a absorção da glicose é mínima, e dez minutos após, a absorção de glicose cresce quinze vezes em relação ao início da atividade, havendo um predomínio de queima de glicose ${ }^{16}$. A literatura apresenta vários estudos mostrando os efeitos benéficos do treinamento aeróbio em indivíduos diabéticos ${ }^{17}$, porém não se encontrou relatos mostrando seus efeitos sobre a força muscular respiratória. Por outro lado, há estudos realizados em outras populações $^{6}$, assim como em diabéticos ${ }^{5}$, mostrando a redução da força muscular inspiratória e os efeitos sobre a mesma após treinamento muscular específico com o uso do equipamento threshold.

Outro parâmetro respiratório avaliado foi a expansibilidade torácica. Sabemos que no decorrer do envelhecimento a parede torácica, assim como a função pulmonar declinam pelo enrijecimento, em virtude de alterações estruturais na caixa torácica, e nas complacências do pulmão e da parede torácica que mudam em direções opostas. Como a força muscular diminui no idoso a capacidade vital e as velocidades do fluxo forçado diminuem. Segundo Jamami et al. ${ }^{18}$, a expansibilidade torácica é definida como a diferença da circunferência a partir da expiração forçada até a inspiração máxima e considera-se um valor normal uma diferença maior que $5 \mathrm{~cm}$. Neste estudo observou-se uma diminuição da expansibilidade torácica antes e após a intervenção, mostrando que os indivíduos já apresentavam valores reduzidos de expansibilidade antes do treinamento e após o exercício aeróbio continuaram com valores abaixo da normalidade, mostrando que o mesmo não exerceu influência sobre esta variável. 
$\mathrm{Na}$ análise da capacidade funcional submáxima, avaliada pelo TC6min observou-se que o treinamento aeróbio promoveu importante aumento da distância percorrida (14\%) e uma diminuição do esforço percebido através da escala de Borg, representando uma melhora da capacidade funcional submáxima. Outros estudos já relatam este benefício em indivíduos diabéticos ${ }^{5}$, portadores de $\mathrm{DPOC}^{19}, \mathrm{ICC}^{6}$, e em outras patologias. A melhora da capacidade funcional submáxima é de grande importância para o indivíduo, pois grande parte das atividades da vida diária são realizadas em níveis submáximos, sendo assim, o TC6min reflete bem a capacidade funcional para as atividades de vida diária ${ }^{20}$. Embora o grupo treinamento aeróbio tenha iniciado com valor basal superior, quando comparado ao grupo controle, em relação a capacidade funcional, isto não interfere na análise, pois em geral, o grupo com pior desempenho apresenta melhores benefícios, o que não foi o caso do grupo G1.

O estudo de Moreira et al. ${ }^{19}$ demonstrou um aumento médio na distância percorrida no TC6min de 76 metros. Os mesmos submeteram 23 indivíduos portadores de DPOC ao treinamento dos membros inferiores durante três meses, três sessões semanais em esteira ergométrica com velocidade $60 \%$ atingida no teste incremental, realizado mensalmente, com inclinação fixa de $6 \%$, velocidade inicial de uma milha por hora, com incrementos de meia milha a cada dois minutos até o máximo tolerado, baseando-se na escala de Borg. No estudo realizado por Dall'Ago et al. ${ }^{6}$, em portadores de ICC, após treinamento muscular inspiratório com threshold durante 3 meses, observaram um aumento de 19\% na distância percorrida.

O treinamento aeróbio exerceu influência positiva também sobre outros parâmetros como a resistência dos membros inferiores e flexibilidade. Sabe-se que o treinamento aeróbio melhora o consumo de oxigênio e isto possibilita que a célula muscular tenha maior disponibilidade de oxigênio por um período maior de tempo e isto favorece uma maior resistência à fadiga através do aumento do limiar anaeróbio ${ }^{21}$. Também foi realizado alongamentos gerais tanto para membros superiores e inferiores antes e após cada sessão e isto contribuiu para a melhora da flexibilidade.
Estudos epidemiológicos e de coorte tem demonstrado forte associação entre obesidade e inatividade física, assim como tem sido relatada associação inversa entre atividade física, índice de massa corpórea (IMC), relação cintura-quadril $(R C / Q)$ e circunferência da cintura. Esses estudos demonstraram que os benefícios da atividade física sobre a obesidade podem ser alcançados com intensidade baixa, moderada ou alta, indicando que a manutenção de um estilo de vida ativo, independente de qual atividade praticada, pode evitar e desenvolvimento desta doença ${ }^{22}$.

Portanto, este estudo mostrou que o treinamento aeróbio foi efetivo na melhora da força muscular ventilatória e capacidade funcional em indivíduos com diabetes mellitus tipo 2. Também, reforça os dados da literatura quanto aos benefícios do treinamento aeróbio em indivíduos com diabetes mellitus, bem como, contribui na produção de conhecimentos quanto, ao dado em que este, também melhora os parâmetros relacionados à força muscular respiratória. A realização de estudos com esta análise é importante para entender melhor a influência da força muscular ventilatória, pois os mecanismos ainda não são muito bem entendidos na literatura. Ainda, o assunto parece não estar esgotado e merece ser mais bem estudado, sugerindo-se uma amostra mais numerosa e a adoção de outras metodologias, a fim de que os mecanismos fisiológicos e fisiopatológicos sejam elucidados, contribuindo com a atuação específica do fisioterapeuta com este tipo de pacientes.

\section{REFERÊNCIAS}

1. Sociedade Brasileira de Diabetes. Diretrizes da Sociedade Brasileira de Diabetes. Tratamento e acompanhamento do diabetes mellitus. 2007 [capturado 2013 Dec 10]; 168 p. Disponível em: http://www.anad.org.br/profissionais/ images/diretrizes_SBD_2007.pdf

2. Ministério da Saúde. Secretaria de Atenção à Saúde. Departamento de Atenção Básica. Diabetes Mellitus: Normas e Manuais Técnicos. Brasília [Internet]. 2006 [capturado 2013 Dec 10]; 56 p. Disponível em: http://bvsms.saude.gov.br/bvs/publicacoes/diabetes_mellit us.PDF

3. DATASUS. Indicadores de mortalidade: taxa de mortalidade por diabetes mellitus. [Internet]. 2006 [capturado 2012 Nov 12] Disponível em: http://tabnet.datasus.gov.br/cgi/idb2006/CapituloC.pdf

4. Meyer FJ, Borst MM, Zugck C, Kirschke A, Schellberg D, 
Kübler W, Haass M. Respiratory muscle dysfunction in congestive heart failure: clinical correlation and prognostic significance. Circulation. 2001;103:2153-8.

5. Wanke $T$, Formanek $D$, Auinger $M$, Popp W, Zwick $H$, Irsigler K. Inspiratory muscle performance and pulmonary function changes in insulin-dependent diabetes mellitus. Am Rev Respir Dis. 1991;143(1):97-100.

6. Dall'Ago P, Chiappa GR, Guths H, Stein R, Ribeiro JP. Inspiratory muscle training in patients with heart failure and inspiratory muscle weakness: a randomized trial. J Am Coll Cardiol. 2006; 47(4):757-63.

7. Borg GAV. Psychophysical bases of perceived exertion. Med Sci Sports Exerc. 1982;14(5):377-81.

8. Ministério da Saúde. Cadernos de Atenção Básica. Departamento da Atenção Básica. Hipertensão arterial sistêmica e diabetes mellitus - protocolo. Brasília [Internet]. 2001 [capturado 2013 Dec 10]; 96 p. Disponível em: $\quad$ http://bvsms.saude.gov.br/bvs/publicacoes/ cd05_06.pdf

9. Ramirez-Sarmiento A1, Orozco-Levi M, Guell R, Barreiro E, Hernandez N, Mota S, Sangenis M, Broquetas JM, Casan P, Gea J. Inspiratory muscle training in patients with chronic obstructive pulmonary disease: structural adaptation and physiologic outcomes. Am J Respir Crit Care Med. 2002;166(11):1491-7.

10. American Thoracic Society. ATS Statement: guidelines for the six-minute walk test. Am J Respir Crit Care Med. 2002; 166:111-7.

11. Perret C, Poiraudeau S, Fermanian J, Colau MM, Benhamou MA, Revel M. Validity, reliability, and responsiveness of the fingertip-to-floor test. Arch Phys Med Rehabil. 2001;82(11):1566-70.

12. Simões RP, Deus APL, Auad MA, Dionísio J, Mazzonetto $M$, Borghi-Silva A. Maximal respiratory pressure in healthy 20 to 89 year-old sedentary individuals of central São Paulo State. Rev Bras Fisioter. 2010;14(1):60-7.

13. Meo SA, Al-Drees AM, Arif M, Shah FA, Al-Rubean K. Assessment of respiratory muscles endurance in diabetic patients. Saudi Med J. 2006;27(2):223-6.

14. Laterza MC, Rondon MUPB, Negrão CE. Efeito anti- hipertensivo do exercício. Rev Bras Hipertens. 2007;14(2):104-11.

15. Winkelmann ER, Chiappa GR, Lima CO, Viecili PR, Stein $\mathrm{R}$, Ribeiro JP. Addition of inspiratory muscle training to aerobic training improves cardiorespiratory responses to exercise in patients with heart failure and inspiratory muscle weakness. Am Heart J. 2009;158(5):768.e1-7.

16. Angus DJ, Febbraio MA, Hargreaves M. Plasma glucose kinetics during prolonged exercise in trained humans when fed carbohydrate. Am J Physiol Endocrinol Metab. 2002;283(3):E573-7.

17. Castaneda C. Type 2 diabetes mellitus and exercise. Rev Nutr Clin Care 2001;3:349-58.

18. Jamami M, Pires VA, Oishi J, Costa D. Efeitos da intervenção fisioterápica na reabilitação pulmonar de pacientes com doença pulmonar obstrutiva crônica (DPOC). Rev Fisioter Univ São Paulo. 1999;6(2):140-53.

19. Moreira MAC, Moraes MR, Tannus R. Teste da caminhada de seis minutos em pacientes com DPOC durante programa de reabilitação. J Pneumol. 2001;27(6):295-300.

20. Barata VF, Gastaldi AC, Mayer AF, Sologuren MJJ. Avaliação das equações de referência para a predição da distância percorrida no teste de caminhada de seis minutos em idosos saudáveis brasileiros. Rev Bras Fisioter. 2005;9(2):165-71.

21. Gademan MG, Swenne CA, Verwey HF, van de Vooren $H$, Haest JC, van Exel HJ, et al. Exercise training increases oxygen uptake efficiency slope in chronic heart failure. Eur J Cardiovasc Prev Rehabil. 2008;15(2):140-4.

22. Ciolac EG, Guimarães GV. Exercício físico e síndrome metabólica. Rev Bras Med Esporte. 2004;10(4):319-24

\section{Endereço para correspondência}

Eliane Roseli Winkelmann

Rua do Comércio $n^{\circ} 3000$

ljuí/RS - CEP 90050-170

Telefone: +55 5533320468

Email: elianew@unijui.edu.b 